\title{
Forty years of AIDS: a retrospective and the way forward
}

\author{
Thomas C. Quinn ${ }^{1,2}$ \\ 'Division of Intramural Research, National Institute of Allergy and Infectious Diseases, NIH, Bethesda, Maryland, USA. 2Division of Infectious Diseases, Johns Hopkins University School of Medicine, Balti- \\ more, Maryland, USA.
}

\section{A historical perspective}

On June 5, 1981, the CDC published a report of five cases of Pneumocystis carinii pneumonia in previously healthy homosexual men living in Los Angeles (1). One month later, the CDC reported more $P$. carinii pneumonia cases and an aggressive form of Kaposi sarcoma in 26 homosexual men residing in California and New York (2). These and other reports of cases associated with opportunistic infections, Kaposi sarcoma, and a very high mortality rate heightened the suspicion of an emerging epidemic of unknown cause that was directly affecting the homosexual male community. However, within a year of these initial reports, it was evident that this disease was not limited to homosexual men and was spreading rapidly into other populations. By July of 1982 the same opportunistic infections and Kaposi sarcoma were being diagnosed in Haitians residing in the United States, individuals with hemophilia, transfusion recipients, individuals using injection drugs, female sex partners of bisexual men with signs of immunodeficiency, and their newborn children (3).

By the end of 1982, there were over 100,000 cases of this mysterious disease in the United States. Cases also started appearing in Europe in the same population groups as in the United States, and in immigrants from sub-Saharan Africa either visiting or residing in Europe (4). By then a clinical case definition had been formed, and the clinical syndrome was labeled "acquired immune deficiency syndrome" (AIDS) to characterize the overwhelming decline in $\mathrm{CD}^{+} \mathrm{T}$ helper lymphocytes with complete loss of cellular immunity and other immunologic abnormalities, resulting in opportunistic infections, cancer, and ultimately death within one year of diagnosis (5). Subsequent investigations in Haiti and several countries in sub-Saharan Africa indicated the broader extent of the disease and its widespread dissemination among heterosexual individuals (6). With the epidemiologic evidence of a widespread fatal sexually transmitted disease that was also transmitted parenterally and perinatally, it was evident that an infectious microbe was responsible, and the hunt for its etiology was on in earnest.

\section{Understanding the etiology and pathogenesis of AIDS}

Unlike our current experience with SARSCoV-2 infection - for which the pathogen was identified, sequenced, and diagnostic tests were made available within a month of clinical recognition - identification of the cause of AIDS took over three years, when French and US investigators identified and cultivated a human retrovirus, labeled "human immunodeficiency virus" (HIV), from infected T cells isolated from patients with $\operatorname{AIDS}(7,8)$. Diagnostic tests took another year to be developed and distributed, allowing the blood supply to be screened and enabling identification of those infected, whether asymptomatic or experiencing opportunistic infections. Once these tests were available, it was clear that this infection had become a pandemic that was spreading faster than previously thought, with over one million estimated infections worldwide by 1986 . We eventually learned that the natural history of this infection from initial exposure to clinical presentation and death typically spanned ten or more years, and that infection in asymptomatic individuals unaware of their infected status con-

Conflict of interest: The author has declared that no conflict of interest exists.

Copyright: () 2021, American Society for Clinical Investigation.

Reference information: J Clin Invest. 2021;131(18):e154196. https://doi.org/10.1172/JCI154196.

tributed to ongoing transmission. Epidemiologic patterns of dissemination could now be better described with improved diagnostic tests, and prevention interventions could be designed, implemented, and monitored for success.

Molecular phylogenetic investigations of HIV followed the cultivation and isolation of the virus from different populations. These studies demonstrated the wide geographic dispersion of the viruses, as well as providing insight into their zoonotic origins (9). Phylogenetic analysis indicated that multiple interspecies transmissions from simian species introduced two genetically distinct types of HIV into the human population: HIV-1, closely related to simian immunodeficiency virus (SIV) from chimpanzees $\left(\mathrm{SIV}_{\mathrm{CPZ}}\right)$; and HIV-2, closely related to SIV from sooty mangabeys $\left(\mathrm{SIV}_{\mathrm{SM}}\right)$. Further studies identified the wide molecular variation in subtypes of HIV-1 and HIV-2, which have different virulence and transmission properties. Molecular segments of the virus were isolated and dissected, and the life cycle of the virus was unraveled with remarkable clarity.

\section{Therapeutic advances}

Tragically, for most of the first decade of the AIDS pandemic, there was essentially no long-lasting effective treatment, and it was a fatal infection associated with prolonged suffering, isolation, discrimination, and stigma. Insights into the viral life cycle led to the development of antiretroviral drugs (ARVs), but it was not until 1997 that the scientific community recognized that a combination of three different drugs including protease inhibitors was required for therapy to be truly effective at prolonging survival by lowering viral load in the peripheral blood (10).

Today, there are more than 30 licensed ARVs, many of which are combined within one tablet, allowing clinicians to treat HIV-infected individuals with one pill, 
A

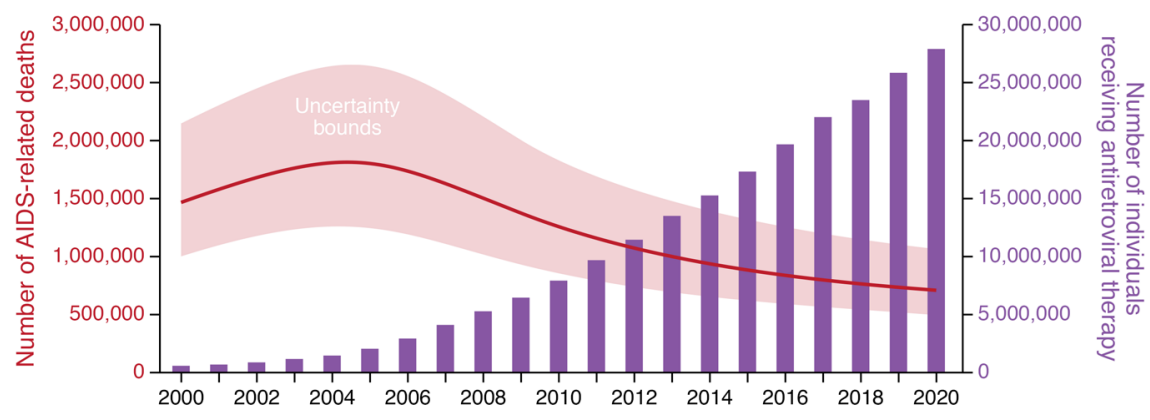

B

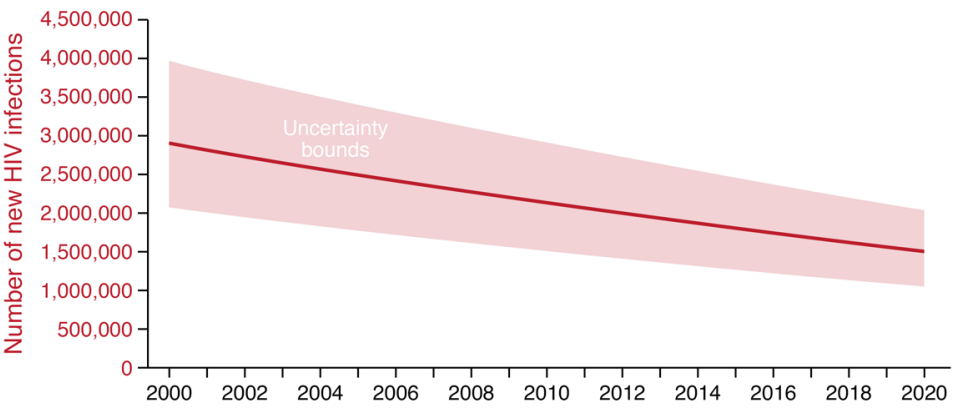

Figure 1. Global statistics for AIDS-related death, HIV treatment, and new HIV infections. (A) Number of AIDS-related deaths and of people receiving HIV treatment globally from 2000 to 2020. The Global Fund was established in 2002 and the President's Emergency Plan for AIDS Relief in 2003, enabling the scale-up of ARVs in the following years, with a reciprocal decline in deaths. (B) Annual number of new HIV infections globally over the same time period. Adapted with permission from UNAIDS (13).

once a day, for life, with an expectation of an average lifespan. Injectable ARVs, such as cabotegravir and rilpivirine, with a long plasma half-life, now enable treatment to be provided as a once-a-month injection (11). These new treatment options offer individualization of care, promote retention in care, and hopefully will avoid the development of resistance. The evolution of treatment regimens will continue, with further studies of potent, long-acting drugs and implantable devices providing even more options to infected patients. Through all of these treatment studies, it became painfully evident that a viral reservoir persisted within well-treated infected individuals that would invariably rebound within weeks to months following cessation of treatment (12). This latent viral reservoir has now become a target for inducing a functional cure, but this goal remains unfulfilled.

Global disparities in patient care By 2000, the lack of access to treatment in many countries highlighted the inequities in care that existed globally. This disparity was most pronounced in resource-limited countries where HIV was most prevalent, as in sub-Saharan Africa. It was not until the third decade of the AIDS pandemic that treatment inequities were truly addressed, first by the United Nations, which formed the Global Fund to Fight AIDS, Tuberculosis, and Malaria in 2002. One year later, the President's Emergency Plan for AIDS Relief (PEPFAR) was launched by President George W. Bush and authorized by the US Congress to bring HIV treatment and prevention to millions of affected individuals. With the establishment of the Global Fund and PEPFAR, 28 million people living with HIV are now in treatment (Figure 1A) (13). While many people with HIV still experience discrimination and need to be engaged in the HIV treatment cascade, the response to AIDS over the last two decades has illustrated that a global effort to address these disparities and inequalities can be effective with intense activism, societal pressures, and adequate financial resources. Together these ini- tiatives embodied the ideals of the global health movement of the 21st century, which include equal access and the provision of high-quality care to all people without discrimination (14).

HIV treatment has been heralded as a success for the field of medicine and a reflection of the scientific advances in therapeutics. However, over the 40 years of the pandemic, an estimated 79 million people became infected, and nearly 40 million people died (13). In 2020 alone, 680,000 people died from AIDS-related illnesses despite the era of antiviral treatment. To address this deficiency in care, the Joint United Nations Programme on HIV/AIDS (UNAIDS) has advanced a new “95-95-95" goal, in which 95\% of infected people globally know of their infection via routine testing; $95 \%$ of those individuals access care and are provided with antiviral drugs; and $95 \%$ of those individuals become virally suppressed by 2025 (15). This treatment cascade serves a dual purpose of increasing survival and limiting viral transmission to others.

\section{Prevention strategies}

Last year, 1.5 million new cases of HIV infection occurred, emphasizing that control and prevention of HIV remain formidable challenges. Prevention in the first two decades was primarily based on education, sexual behavioral changes, use of condoms, blood screening, and needle exchange programs. While these prevention efforts reduced transmission, their cumulative effects were limited, as evidenced by the 2-3 million new infections occurring each year during the second decade of this disease. Much work in the last two decades demonstrated the additive effects of biomedical prevention to behavioral and structural interventions. The concept of "treatment as prevention" was fully endorsed following the pivotal HIV Prevention Trials Network (HPTN) 052 studies, which demonstrated a $96 \%$ efficacy in prevention of transmission among discordant couples if the infected person was in treatment and virally suppressed (16). Perinatal transmission from an infected mother on treatment with ARVs also reduced HIV transmission by over $90 \%$, raising the hope of an AIDSfree generation for children. Multiple population-based studies demonstrated 
universally that in individuals with undetectable viral loads due to ARV treatment, HIV is untransmissible (Undetectable Equals Untransmittable, or $\mathrm{U}=\mathrm{U})$. This dictum led to the recommendation by all advisory bodies to start treatment as early as possible to achieve maximum sustained viral suppression, thereby decreasing transmission (Figure 1B) (17).

The use of antiviral drugs to prevent transmission also influenced a series of double-blind placebo-controlled studies that demonstrated the high efficacy of preexposure prophylaxis (PrEP) with select ARVs to prevent acquisition among HIV-negative people $(18,19)$. As for treatments, a range of PrEP regimens have evolved, from a daily single oral tablet, "on-demand" prophylaxis, vaginal rings containing ARVs, and long-acting injectable ARVs and implant devices containing ARVs and contraceptives. Coincident with these PrEP studies, three randomized studies in sub-Saharan Africa also demonstrated that voluntary male circumcision prevented HIV acquisition in heterosexual men by $50 \%-60 \%$, with a subsequent community effectiveness of over $70 \%$ (20). This single, one-time surgical procedure was endorsed and recommended by the WHO, and to date, 18 million young African males have been circumcised, thereby averting millions of infections. Finally, in efforts to control HIV globally, many societies are combining all these interventions to prevent HIV infection while providing universal treatment to those already infected to more effectively limit both HIV transmission and acquisition. While successes in combination HIV prevention have now been reported (21), stubborn obstacles such as denialism, stigma, and discrimination have prevented its full endorsement in populations that continue to have a high incidence, as in some eastern European countries.

Ultimately, the control of HIV/AIDS would benefit from the development and implementation of a highly efficacious HIV vaccine. Unfortunately, this has been an elusive goal, with only partial success in one trial (22) and many disappointments from trials incorporating different vaccine constructs. However, the scientific community remains undeterred, and advances in the field of vaccine science may eventually yield a preventative vaccine.
Until then, global control efforts have incorporated aggressive testing programs to help people know their infection status. Early treatment with intensive linkage and retention in care are offered to infected individuals to reduce morbidity and subsequent transmissions $(13,15)$. For HIV-uninfected individuals, comprehensive prevention interventions are offered, incorporating educational programs (particularly for young girls), condom distribution, PrEP for high-risk individuals, voluntary male circumcision, and, where necessary, needle exchange programs. Global and individual-country goals have been enumerated to provide the necessary road map to achieve success. The goal of the 2020 federal program "Ending the HIV Epidemic in the U.S." is to reduce new infections by $75 \%$ over five years and by $90 \%$ by 2030 (23). UNAIDS has set similar global goals, but all these initiatives require a comprehensive and effective implementation of the above interventions, while simultaneously addressing the inherent inequities in care.

\section{Conclusions}

Over the 40 years of the AIDS pandemic, many scientific discoveries have changed the course of this tragic disease. Sadly, too many people have died, and too many children have been orphaned by the premature death of their parents. The economic, political, and societal impact of AIDS over the past 40 years is incalculable. On the positive side, this global pandemic has in many ways resulted in major scientific discoveries relevant to advancing the fields of virology, immunology, epidemiology, public health, molecular biology, and antiviral therapeutics. The painful lessons and scientific advances gained over the years from the AIDS pandemic have influenced our response to the current SARS-CoV-2 pandemic as well as other emerging diseases. HIV/AIDS has broadened our perspectives on health in general, highlighting both the disparities and inequities in access to treatment and care as well as the knowledge of how to treat those in need more effectively. While AIDS is recognized as a clinical condition, we have also learned the perils of societally inflicted social isolation, discrimination, and unrelenting stigma. It will be how we address the totality of these issues, especially when armed with our current and future scientific advances, that will truly write the long history of this disease.

Address correspondence to: Thomas C. Quinn, Rangos Bldg, Room 531, 855 N. Wolfe Street., Baltimore, Maryland 21205, USA. Phone: 410.955.7635; Email: tquinn2@jhmi.edu.

1. Centers for Disease Control. Pneumocystis pneumonia-Los Angeles. MMWR Morb Mortal Wkly Rep. 1981;30(21):250-252.

2. Centers for Disease Control. Kaposi's sarcoma and Pneumocystis pneumonia among homosexual men - New York City and California. MMWR Morb Mortal Wkly Rep. 1981;30(25):305-308.

3. Centers for Disease Control. Update on acquired immune deficiency syndrome (AIDS)-United States. MMWR Morb Mortal Wkly Rep. 1982;31(37):507-508.

4. Clumeck N, et al. Acquired immunodeficiency syndrome in African patients. $N$ Engl J Med. 1984;310(8):492-497.

5. Fauci AS, et al. NIH conference. Acquired immunodeficiency syndrome: epidemiologic, clinical, immunologic, and therapeutic considerations. Ann Intern Med. 1984;100(1):92-106.

6. Quinn TC, et al. AIDS in Africa: an epidemiologic paradigm. Science. 1986;234(4779):955-963.

7. Barré-Sinoussi F, et al. Isolation of a T-lymphotropic retrovirus from a patient at risk for acquired immune deficiency syndrome (AIDS). Science. 1983;220(4599):868-871.

8. Gallo RC, et al. Frequent detection and isolation of cytopathic retroviruses (HTLV-III) from patients with AIDS and at risk for AIDS. Science. 1984;224(4648):500-503

9. Hahn $\mathrm{BH}$, et al. AIDS as a zoonosis: scientific and public health implications. Science. 2000;287(5453):607-614

10. Gulick RM, et al. Treatment with indinavir, zidovudine, and lamivudine in adults with human immunodeficiency virus infection and prior antiretroviral therapy. N Engl J Med. 1997;337(11):734-739.

11. Swindells S, et al. Long-acting cabotegravir and rilpivirine for maintenance of HIV-1 suppression. $N$ Engl J Med. 2020;382(12):1112-1123.

12. Finzi D, et al. Latent infection of CD $4+\mathrm{T}$ cells provides a mechanism for lifelong persistence of HIV-1, even in patients on effective combination therapy. Nat Med.1999;5(5):512-517.

13. UNAIDS. Global AIDS Update. Confronting Inequalities. Lessons for pandemic responses from 40 years of AIDS. https://www.unaids. org/sites/default/files/media_asset/2021global-aids-update_en.pdf. Accessed August 17, 2021.

14. Piot P, Quinn TC. Response to the AIDS pandemic - a global health model. N Engl JMed. 2013;368(23):2210-2218.

15. UNAIDS. On the Fast Track to an AIDS free generation. https://www.unaids.org/sites/default/ files/media_asset/GlobalPlan2016_en.pdf. 
Accessed August 17, 2021.

16. Cohen MS, et al. Prevention of HIV-1 infection with early antiretroviral therapy. N Engl JMed. 2011;365(6):493-505.

17. Martin TCS, et al. Rapid antiretroviral therapy among individuals with acute and early HIV. Clin Infect Dis. 2021;73(1):130-133.

18. Baeten JM, et al. Antiretroviral prophylaxis for HIV prevention in heterosexual men and women. N Engl JMed. 2012;367(5):399-410. 19. Molina J-M, et al. ANRS IPERGAY Study Group. On-demand preexposure prophylaxis in men at high risk for HIV-1 infection. $N$ Engl J Med. 2015;373(23):2237-2246.

20. Gray R, et al. The effectiveness of male circumcision for HIV prevention and effects on risk behaviors in a posttrial follow-up study. AIDS. 2012;26(5):609-615.
21. Grabowski MK, et al. Rakai Health Sciences Program. HIV prevention efforts and incidence of HIV in Uganda. N Engl JMed. 2017;377(22):2154-2166.

22. Rerks-Ngarm S, et al. MOPH-TAVEG investigators. Vaccination with ALVAC and AIDSVAX to prevent HIV-1 infection in Thailand. $N$ Engl J Med.2009;361(23):2209-2220.

23. Fauci AS, et al. Ending the HIV epidemic: a plan for the United States. JAMA. 2019;321(9):844-845. 